\title{
DESFRALDE INFANTIL E TREINAMENTO ESFINCTERIANO: UMA REVISÃO DE
} LITERATURA

\author{
Rejane Cassol'; Regina Celia de Castro Gomes²; Tainá Ribas de Moraes ${ }^{3}$ \\ Rosane Gomes de Oliveira ${ }^{4}$; Silvia Naujork ${ }^{5}$; Pedro José Prá6; Nadiesca Taisa \\ Filippin $^{7}$; Josiane Lieberknecht Wathier Abaid ${ }^{8}$
}

\section{RESUMO}

Esse artigo teve o objetivo de investigar como vem sendo abordados na literatura o desfralde infantil e o treinamento esfincteriano. O aprendizado do controle esfincteriano é influenciado por fatores fisiológicos, psicológicos e socioculturais. Trata-se de uma revisão narrativa de literatura. Foram realizadas buscas no mês de agosto e setembro nas bases de dados: Google Acadêmico e SCIELO. Após as buscas e aplicação dos filtros, encontrou-se cinco documentos, sendo dois com as palavras-chaves: "desfralde infantil" e três com as palavras-chaves: "treinamento esfincteriano". Entendeu-se que o processo de desfralde ou treinamento esfincteriano pode desencadear momentos de ansiedade tanto para os pais quanto para as crianças e algumas vezes essa ansiedade acaba por atrapalhar ou retardar o processo.

Palavras-chave: Desfralde, Esfincteriano, Infância.

Eixo Temático: Atenção Integral e Promoção à Saúde.

\section{INTRODUÇÃO}

As questões relativas à linguagem e ao pensamento no ser humano sempre foram objeto de indagações e perplexidades. Nas últimas décadas essas questões tiveram avanços em novas direções, ligadas sobretudo a conexões com a evolução

\footnotetext{
${ }_{1}^{1}$ Aluna Pós-Graduação em Saúde materno infantil UFN - Apresentadora: rejanecassol@hotmail.com

2 Aluna Pós-Graduação em Saúde materno infantil UFN - taina.ribas@ufn.edu.br

${ }^{3}$ Aluna Pós-Graduação em Saúde materno infantil UFN - rcgomes966@gmail.com

${ }^{4}$ Aluna Pós-Graduação em Saúde materno infantil UFN - rosane.oliveira@ufn.edu.br

5 Aluna Pós-Graduação em Saúde materno infantil UFN - silvianaujorks@gmail.com

${ }^{6}$ Aluno Pós-Graduação em Saúde materno infantil UFN - pedropra@terra.com.br

${ }^{7}$ Docente do Mestrado Profissional em Saúde Materno Infantil - nadifilippin@edu.br josianelieb@edu.br

${ }^{8}$ Docente do Mestrado Profissional em Saúde Materno Infantil - josianelieb@edu.br
} 
biológica. Nos primeiros anos da criança, os circuitos neurais do cérebro são formados e fortalecidos por meio de estímulos e relações de vínculo, estabelecem as bases para o desenvolvimento ao longo da vida. A saúde física e emocional, habilidades sociais e as capacidades cognitivo-linguísticas emergem nessa fase e são pré-requisitos importantes para o sucesso na escola, na vida social e nas suas necessidades fisiológicas (SHONKOFF; BOYCE; MCEWEN, 2009).

A criança, como todo ser humano, está inserida em um contexto social e histórico, sofrendo influências da sociedade e apropriando-se de valores e comportamentos próprios de seu tempo e lugar. A criança é a pessoa, o cidadão com direitos, e deve ser considerada um ator social, sujeito de seu processo de socialização, um consumidor com poder, um indivíduo emancipado em formação, isto é, que está aprendendo a exercer seus direitos (BELLONI, 2009; COHEN, 2021). O aprendizado do controle esfincteriano é influenciado por fatores fisiológicos, psicológicos e socioculturais. A família é o primeiro referencial da criança, seguida pelo ingresso na escola (MOTA, et al., 2010). Na área do desenvolvimento infantil as maiores preocupações estão relacionadas ao treinamento de esfíncteres e os seus distúrbios (HOWE; WALKER, 1992).

Antes dos dois anos de vida o corpo da criança não está desenvolvido suficiente para controlar sua vontade de fazer xixi ou cocô, portanto o uso de fralda é necessário. Após isso já é possível começar o processo do desfralde diurno, que leva pelo menos 6 meses para se consolidar. treinamento esfincteriano pode gerar conflitos e ansiedade, com possível impacto negativo na relação pais-criança e complicações como recusa do treinamento esfincteriano, constipação e encoprese (BRAZELTON; 1962, TAUBMAN; BUZBY; 1997). O treinamento esfincteriano pode gerar conflitos e ansiedade, com possível impacto negativo na relação pais-criança e complicações como recusa, constipação e encoprese (BRAZELTON; 1962, FISHMAN, et al., 2002). A Academia Americana de Pediatria publicou, em 1998, as primeiras diretrizes sobre os métodos de treinamento e, em 2003, um guia de treinamento esfincteriano escrito por pediatras, que aborda métodos de treinamento, dúvidas e dificuldades mais frequentes (AAP, 1998, WOLRAICH; 2003).

Nesse sentido, esse artigo tem o objetivo de investigar como vem sendo 
abordadas na literatura o desfralde infantil e o treinamento esfincteriano.

\section{METODOLOGIA}

Trata-se de uma revisão narrativa de literatura. Tal revisão é constituída por uma análise ampla da literatura, sem estabelecer uma metodologia rigorosa e replicável em nível de reprodução de dados e respostas quantitativas para questões específicas (VOSGERAU; ROMANOWSK, 2014). A revisão narrativa é de suma importância para a aquisição e atualização do conhecimento sobre uma temática específica, evidenciando novas ideias, métodos e subtemas que têm recebido maior ou menor ênfase na literatura selecionada (ELIAS et al., 2012). A palavra-chave "desfralde infantil" foi a primeira a ser pesquisada. Após a análise dos artigos encontrados na base de dados, optou-se por incluir a busca por "treinamento esfincteriano" para entender melhor sobre a literatura disponível sobre o tema.

Para chegar ao objetivo proposto foram realizadas buscas no mês de agosto e setembro do presente ano nas bases de dados: Google Acadêmico e SCIELO. A estratégia de busca no Google Acadêmico obedeceu ao seguinte fluxograma (Figura $1)$.

Figura 1: Fluxograma de busca no Google acadêmico.

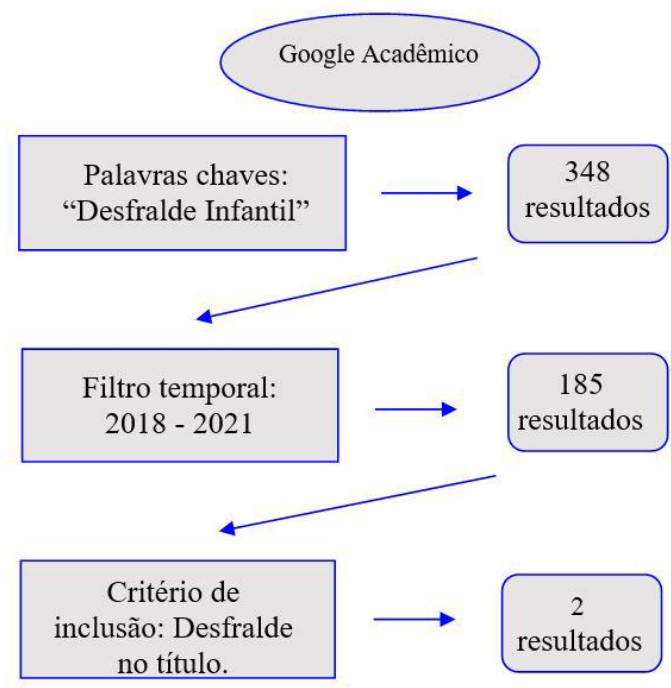




\section{QUFN}

A estratégia de busca na base de dados SCIELO obedeceu ao fluxograma na figura 2.

Figura 2: Fluxograma de busca na base SCIELO.

\section{SCIELO}

Palavras-chaves:

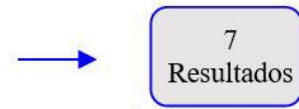

Critérios de exclusão:

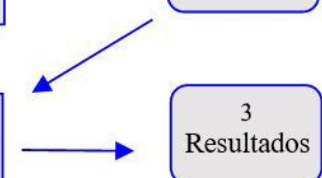

Após as buscas e aplicação dos filtros, resultaram em 5 documentos, sendo 2 com as palavras-chaves: "desfralde infantil" e 3 com as palavras-chaves: "treinamento esfincteriano". A síntese da extração dos dados é apresentada nos resultados e discussões.

\section{RESULTADOS E DISCUSSÕES}

A busca de documentos que compuseram esse estudo, identificou cinco referências sobre o desfralde infantil e o treinamento esfincteriano. Com o resultado selecionados fez-se a leitura e extração dos dados (Quadro 1) principais para compreender a essência de cada trabalho. 
Quadro 1 - Extração dos dados.

\begin{tabular}{|c|c|c|c|}
\hline TíTULO & AUTORES & ANO & MÉTODO \\
\hline $\begin{array}{l}\text { O } \\
\text { entendimento } \\
\text { de } \\
\text { professoras e } \\
\text { familiares } \\
\text { referente ao } \\
\text { processo de } \\
\text { desfralde de } \\
\text { crianças bem } \\
\text { pequenas. }\end{array}$ & COHEN, R. A. S. & 2021 & $\begin{array}{l}\text { Abordagem } \\
\text { qualitativa com } \\
\text { técnica de } \\
\text { produção de dados } \\
\text { por questionário. }\end{array}$ \\
\hline $\begin{array}{ll}\text { Estudo da } \\
\text { associação } \\
\text { entre } & \\
\text { desfralde e } & 0 \\
\text { uso do eu. } & \end{array}$ & $\begin{array}{l}\text { MORAES, A. V. } \\
\text { P. M.; LERNER, } \\
\text { R.; AMARAL, L. } \\
\text { Q. }\end{array}$ & 2020 & $\begin{array}{lr}\text { Abordagem } & \\
\text { qualitativa } & \text { com } \\
\text { observação } & \text { e } \\
\text { questionário, } \\
\text { durante } & \text { um } \\
\text { período de } & 10 \\
\text { meses. } & \end{array}$ \\
\hline
\end{tabular}

\author{
MIRANDA, J. E. 2011 \\ G. \\ B.; \\ MACHADO, N \\ C.
}

Estudo de coorte transversal em crianças sadias, aplicando-se um questionário estruturado para 100 responsáveis por crianças entre três e seis anos de idade.

$\begin{array}{ll}\text { Avaliação } & \text { MOTA, D. M.; } \\ \text { longitudinal } & \text { BARROS, A. J. } \\ \text { do controle } & \text { D.; } \\ \text { esfincteriano } & \text { MATIJASEVICH, } \\ \text { em uma } & \text { A.; SANTOS, I. S. } \\ \text { coorte de } & \\ \text { crianças } & \\ \text { Brasileiras } & \end{array}$

\author{
4.231 crianças \\ nascidas no ano de \\ 2004, estudo \\ longitudinal. Em \\ visitas domiciliares \\ realizadas aos 12 , \\ 24 e 48 meses, as \\ mães \\ responderam a um \\ questionário com \\ questões \\ sociodemográficas \\ características dos \\ hábitos miccionais \\ e intestinais das \\ crianças, com \\ atenção ao
}

\section{RESULTADOS}

O desfralde deve-se levar em consideração 0 respeito ao tempo da criança, importante é perceber a criança como um ser único que possui direitos e seu próprio tempo para se conhecer e se desenvolver em sua plenitude.

Os resultados indicaram associação

entre desfralde e uso do "eu" principalmente

nos seguintes aspectos:

predomínio do uso

do "eu" para fazer referência a si mesmo, uso

do "eu" em situações de independência e em

situações nas quais há o desejo de mostrar às outras pessoas habilidades já adquiridas.

97\% das crianças foram treinadas em casa pelas mães e $92 \%$ delas utilizaram a intuição, a experiência com o filho anterior e o aprendizado com as avós. O treinamento do controle esfincteriano anal e vesical foi iniciado simultaneamente em $84 \%$ dos casos, sendo o controle anal adquirido primeiramente em $41 \%$ das crianças.

Aos 48 meses, a maioria das crianças estava sem fraldas durante o dia $(98,5 \%)$ e à noite (83\%), sem diferença entre os sexos. A idade média de início de treinamento esfincteriano foi 22 meses, com início mais precoce nas meninas. A duração média do treinamento foi de 3,2 meses, sem diferença entre os sexos. 
treinamento

esfincteriano.

\begin{tabular}{|c|c|c|c|}
\hline $\begin{array}{l}\text { Treinamento } \\
\text { esfincteriano: } \\
\text { métodos, } \\
\text { expectativas } \\
\text { dos pais e } \\
\text { morbidades } \\
\text { associadas. }\end{array}$ & $\begin{array}{l}\text { MOTA, D. M.; } 2008 \\
\text { BARROS, A. J. } \\
\text { D.; }\end{array}$ & $\begin{array}{lr}\text { Revisão } & \text { de } \\
\text { literatura científica } \\
\text { e leiga sobre o } \\
\text { treinamento } \\
\text { esfincteriano, } \\
\text { abordando } \\
\text { expectativas dos } \\
\text { pais, métodos } \\
\text { disponíveis para } \\
\text { aquisição r do } \\
\text { controle r } \\
\text { esfincteriano } \\
\text { morbidades } \\
\text { associadas. }\end{array}$ & $\begin{array}{l}\text { Os pais apresentam } \\
\text { expectativas irreais sobre } \\
\text { idade de retirada de fraldas, } \\
\text { sem levar em conta o } \\
\text { desenvolvimento infantil. As } \\
\text { estratégias de treinamento } \\
\text { não se modificaram nas } \\
\text { últimas décadas. O início } \\
\text { precoce do treinamento } \\
\text { esfincteriano e eventos } \\
\text { estressantes durante o } \\
\text { período podem prolongar o } \\
\text { processo de treinamento. }\end{array}$ \\
\hline
\end{tabular}

Org.: Os autores

A partir da leitura dos textos selecionados, foi possível observar que os estudos são unânimes sobre o tempo e as particularidades de cada criança. Cohen (2021) coloca que é preciso levar em consideração que é a criança quem deve estar pronta para o momento do desfralde e cabe aos adultos ficarem atentos e darem o suporte necessário. Ressalta-se ainda que a aprendizagem e o controle dos esfíncteres são grandes desafios cognitivo, social e motor para a criança (BARBOSA; QUADROS, 2017). Cohen (2021) observou que algumas famílias usaram estratégias como o uso de troninhos e penicos para introduzir junto a rotina do banheiro, dessa forma familiarizando a criança com o formato e modo de realizar suas necessidades.

É importante observar que existem inúmeras formas de incentivar a criança ao desfralde, as possibilidades podem ser das mais simples como contar uma história como mais elaborada com troninhos musicais entre outros modos. Assim como é preciso estratégias para dar suporte no processo de deixar as fraldas, existem elementos muito importantes que podem auxiliar na segurança da criança e na participação da família (COHEN, 2021).

Moraes et al. (2020) trazem à luz, a respeito do desfralde, o uso do "eu", ao começar a falar, a criança faz referência a si mesma na terceira pessoa, imitando a forma como os adultos se referem a ela. Chega, porém, um momento em que ela começa a dizer "eu" ao se referir a ela mesma. De acordo com Mota; Barros (2008), o desfralde se estabelece, na maioria das culturas, entre 24 e 36 meses. Tais eventos, 
acontecem de modo independentes, mas na mesma época, tipicamente entre 2 e 3 anos de idade.

A pesquisa de MORAES et al., (2020) observou e corroborou com a literatura existente que evidenciam o desfralde mais precoce em meninas do que em meninos, porém tais dados precisam ser melhor observados (BRAZELTON, 1962). Shum et al., (2001) demonstraram 11 sinais em seu estudo, que indicam a prontidão para o treinamento do desfralde aos 24 meses, as meninas dominaram somente dois e os meninos não eram proficientes em alguns sinais, sendo a mediana da idade para início do treinamento de 25,5 meses para as meninas e 30,5 meses para os meninos. Por outro lado, no estudo de Moraes et al, (2020, p.120) "o fator "idade cronológica" não pareceu ser preponderante na conquista do desfralde". A idade cronológica é considerada um "momento mágico" para iniciar ou não a retirada das fraldas, sendo que o início antes dos 24 meses, especialmente antes dos 18 meses, é a maior preferência (RITBLATT, et al., 2003).

No estudo de Miranda; Machado (2012) foram avaliadas crianças de 3 a 6 anos de idades, a respeito de seu controle esfincteriano. O estudo evidenciou que o treinamento do controle esfincteriano anal e vesical foi iniciado simultaneamente em $84 \%$ dos casos, sendo o controle anal adquirido primeiramente em $41 \%$ das crianças. Ainda foi possível observar que não houve aumento significativo de constipação intestinal após o início do treinamento e não foram observados casos de encoprese.

No estudo de Blum et al. (2004), os autores observaram que a constipação se relaciona mais à recusa de usar o banheiro do que à consequência do treinamento. Salienta-se que o estudo observou a classe econômica das famílias as quais participaram do estudo, sendo que as de baixa renda tiveram o fator custo das fraldas como determinantes para iniciar o treinamento.

Pachter; Dworkin (1997), observam que maioria dos pais, mesmo os mais informados, apresentam expectativas inadequadas em relação à idade em que o controle esfincteriano é adquirido. Miranda; Machado (2012), trazem em seu estudo a discussão sobre disfunção miccional, constipação, recusa em ir ao banheiro e encoprese. Foi observado um aumento sobre a prevalência da disfunção miccional, muito embora as causas ainda sejam pouco conhecidas. 
Observou-se que tal disfunção é vista como normal pelos pais, pois eles consideram fazer parte do comportamento das crianças. Ainda é importante observar a posição anatômica da criança na hora da micção, o esfíncter uretral, tem um importante papel nas disfunções miccionais na infância. Tais disfunções ocorrem quando é utilizado um penico muito baixo, criando uma posição de "cócoras", que estimula pressão durante a micção ou ainda, um vaso sanitário normal, sem redutor, motiva que a criança contraia os músculos da coxa e não relaxe a musculatura perineal, dificultando o esvaziamento vesical (CHRISTOPHERSEN, 1991; VERNON et al., 2003; VASCONCELOS et al., 2006). De Paepe et al., (2002) observam que com a repetição de tal situação acaba por promover um fluxo retrógrado de bactérias da uretra para a bexiga, causando as infecções urinárias de repetição.

No caso das constipações os autores observaram que há prevalências inversas a faixa etária na infância, somada a outros fatores como mudança de dieta, genética, dor ou dificuldade para evacuar, o treinamento esfincteriano inadequado pode ser traumático e causar constipação (BAKER et al., 1999).

As recusas para o treinamento esfincteriano mostraram-se na maioria dos casos como o maior agente de negação a dor ao evacuar em crianças menores de 2 anos de idade, após os 2 anos, o mesmo motivo ainda foi o mais citado. Outras causas como a vergonha também aparecem na literatura, como em Taubman et al. (2003), pois a conotação negativa relacionada às fezes presente na nossa cultura pode levar as crianças a terem vergonha de suas fezes, escondendo-se para eliminá-las, sem a presença de um adulto.

\section{CONCLUSÃO}

O desfralde infantil e o treinamento esfincteriano são fortemente influenciados por várias culturas, sendo um grande marco no desenvolvimento infantil. Não há na literatura uma unanimidade na idade cronológica para esse evento, pois cada cultura tem um método e cada criança é um ser único que deve ser respeitado.

É importante salientar que o desfralde representa um desafio para a criança adquirir parte da sua independência. Existem orientações e diretrizes existentes para 
que o treinamento esfincteriano seja feito de forma harmoniosa e sem prejuízo para as crianças.

Os estudos demonstraram que apesar de ser assunto de grande discussão na academia, o desfralde infantil como palavra-chave encontrou poucos trabalhos com esse título, sendo necessária a pesquisa com treinamento esfincteriano. O desfralde infantil nos demais artigos da busca, aparecia apenas como tema secundário nos documentos encontrados.

Entendeu-se ainda que o processo de desfralde ou treinamento esfincteriano é um processo de ansiedade tanto para os pais quanto para as crianças e algumas vezes essa ansiedade acaba por atrapalhar ou retardar o processo. Por ser um processor muito importante de descoberta e conquista da autonomia da criança, é importante não apressar, mas sim respeitá-la como um ser único.

\section{REFERÊNCIAS}

BAKER SS, LIPTAK GS, COLLETTI RB, CROFFIE JM, DI LORENZO C, ECTOR W, et al. Constipation in infants and children: evaluation and treatment. A medical position statement of the North American Society for Pediatric Gastroenterology and Nutrition. J Pediatr Gastroenterol Nutr. 1999.

BELLONI, M. L. O que é sociologia da infância. Campinas: Acadêmico de bolso, 2009.

BLUM NJ, TAUBMAN B, NEMETH N. Relationship between age at initiation of toilet training and duration of training: a prospective study. Pediatrics. 2003.

BRAZELTON TB. A child-oriented approach to toilet training. Pediatrics. 1962.

COHEN, R. A. S. O entendimento de professoras e familiares referente ao processo de desfralde de crianças bem pequenas. Trabalho de Conclusão de Curso: Universidade Federal de Santa Maria. 2021.

DE PAEPE H, HOEBEKE P, RENSON C, VAN LAECKE E, RAES A, VAN HOECKE $E$, et al. Pelvic-floor therapy in girls with recurrent urinary tract infections and dysfunctional voiding. Br J Urol. 1998.

HOWE AC, WALKER CE. Behavioral management of toilet training, enuresis, and encopresis. Pediatr Clin North Am. 1992. 
MIRANDA, J. E. G. B.; MACHADO, N. C. Treinamento esfincteriano anal: estudo transversal em crianças de 3 a 6 anos de idade. Rev. paul. pediatr. 2011.

MORAES, A. V. P. M.; LERNER, R.; AMARAL, L. Q. Estudo da associação entre o desfralde e o uso do eu. A Psicologia em suas Diversas Áreas de Atuação. 2020.

MOTA, D. M.; BARROS, A. J. D. Treinamento esfincteriano: métodos, expectativas dos pais e morbidades associadas. J. Pediatr. V. 29. n. 3. 2011.

MOTA, D. M.; BARROS, A. J. D.; MATIJASEVICH, A.; SANTOS, I. S. Avaliação longitudinal do controle esfincteriano em uma coorte de crianças Brasileiras. J. Pediatr.v. 85 n. 5. 2010.

PACHTER LM, DWORKIN PH. Maternal expectations about normal child development in 4 cultural groups. Arch Pediatr Adolesc Med. 1997.

SCHUM TR, MCAULIFFE TL, SIMMS MD, WALTER JA, LEWIS M, PUPP R. Factors associated with toilet training in the 1990s. Ambul Pediatr. 2001.

SHONKOFF, J.P., BOYCE, W.T., MCEWEN, B.S. Neuroscience, molecularbiology and the childhood roots of health disparities: buildinga new framework for health promotion and disease prevention.JAMA. 2009.

TAUBMAN B. Toilet training and toileting refusal for stool only: a prospective study. Pediatrics. 1997. 